\title{
Proton structure, its spin and tensor gluons.
}

\author{
Spyros Konitopoulos ${ }^{1, \star}$ and George Savvidy ${ }^{1, \star \star}$ \\ ${ }^{1}$ Demokritos National Research Center, Ag. Paraskevi, Athens, Greece
}

\begin{abstract}
We assume that inside the hadrons there are additional partons - tensorgluons which carry the same charges as the gluons, but have larger spin. That assumption leads to a possible contribution of polarised tensorgluon density to the spin of the nucleon. The nonzero density of tensorgluons can be created inside a nucleon by radiation of tensorgluons by gluons. Tensorgluons can carry a part of nucleon momentum together with gluons. Because tensorgluons have a larger spin, they can influence the spin structure of the nucleon. This contribution appears in the next to leading order, compared to the gluons and can provide a substantial screening effect due to the larger spin of tensorgluons.
\end{abstract}

\section{Introduction}

We assume that inside the hadrons there are additional partons - tensorgluons - which carry the same charges as the gluons, but have larger spin $[1,2]$. That assumption leads to a possible contribution of polarised tensorgluon density to the spin of the nucleon. The nonzero density of tensorgluons can be created inside a nucleon by radiation of tensorgluons by gluons. The tensorgluons can carry a part of nucleon momentum together with gluons. Because tensorgluons have larger a spin $s(s=2,3, \ldots)$, they can influence the spin structure of the nucleon. Our aim is to analyse a possible contribution of polarised tensorgluon density $\Delta T_{s}$ into the spin of the nucleon, if they are present in the nucleon.

The spin structure of the nucleon remains the essential problem of nonperturbative QCD and hadronic physics. One of its most significant manifestations is the so-called spin crisis, or spin puzzle, related to the surprisingly small fraction of proton polarisation carried by the quarks [3-10]. The contribution from the quarks spin is found out to be small, approximately $25 \%$ of the total proton spin, and it is expected that the rest should come from the spin of gluons, quark sea polarisation and the orbital angular momentum of quarks and gluons. This problem attracted the attention to the peculiarities of the underlying QCD description of the nucleon spin, and, in particular, to the role of the gluons [17-22, 26]. One of the most interesting suggestions to come out of the spin crisis was that gluon polarisation $\Delta G$ may contribute significantly to the nucleon spin [20-23]. The amount of gluon polarisation from the experiment is approximately $20 \%[6,11,12,15,16]$ and it is not by itself sufficient to resolve the problem. It remains to identify the rest $(50 \%)$ of the nucleon spin. The experimental indications of the small value of the polarisation carried by the quarks and gluons point to a possible contribution of the orbital angular momentum of the constituent patrons or to a possible contribution coming from the polarisation of additional kind of partons in the nucleon.

\footnotetext{
$\star$ e-mail: spykoni@inp.demokritos.gr

$\star \star$ e-mail: savvidy@inp.demokritos.gr
} 


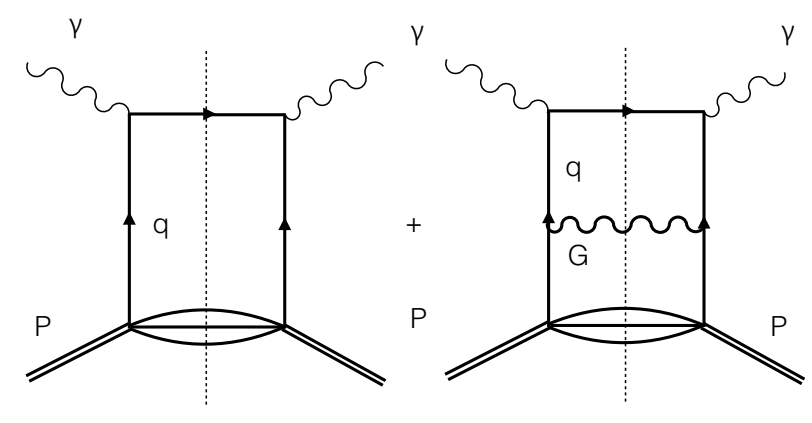

Figure 1. Scattering of a photon on a proton quark $q$ in the leading and next to leading order through the gluon exchange.

Following the suggestion [1,2] that there are additional patrons in the nucleon, one can propose that the nucleon's spin is accounted by the quark spin $\frac{1}{2} \Delta \Sigma$, gluon spin $1 \Delta G$, as well as by the tensorgluon spin $s \Delta T_{s}$ and the total orbital angular momentum $L_{z}$. Physically the quantities $\Delta \Sigma, \Delta G, \Delta T_{s}$ represent the differences between the quark number densities, gluons and tensorgluons with spin parallel to the nucleon spin and those with spin anti-parallel (20). Because the spin of the nucleon is $\frac{1}{2} \hbar$, one should have the following spin sum rule of helicity weighted distributions:

$$
\frac{1}{2} \Delta \Sigma+\Delta G+\sum_{s} s \Delta T_{s}+L_{z}=\frac{1}{2}
$$

In this equation the helicity of the gluon is equal to one, of the tensorgluons is $s=2,3, .$. and the summation is over all "active" tensorgluons in the nucleon. The various components of the nucleon spin in the above equation can be measured experimentally $[6,8-10,12,14-16]$. Our aim is to consider a possible contribution of tensorgluons of spin $\mathrm{s}$, the $s \Delta T_{s}$ contribution.

Considering the influence of polarised tensorgluons density $\Delta T_{s}$ to the singlet part of the first moment $g_{1}$ in (6) we found an additional contribution from tensorgluons of spin s:

$$
\begin{aligned}
I_{0}=\frac{1}{9} & \left(\Delta \Sigma-n_{f} \frac{\alpha\left(Q^{2}\right)}{2 \pi} \Delta G\right)\left(1-\frac{\alpha\left(Q^{2}\right)}{2 \pi} \frac{3\left(12 s^{2}-1\right)-8 n_{f}}{3\left(12 s^{2}-1\right)-2 n_{f}}\right)+ \\
& +n_{f} \frac{\alpha\left(Q^{2}\right)}{2 \pi} \Delta T_{s} \frac{\sum_{k=1}^{2 s+1} \frac{1}{k}}{3\left(12 s^{2}-1\right)-2 n_{f}} .
\end{aligned}
$$

The present paper is organized as follows. In section 2 the basic formulae for polarised electroproduction are recalled and short review of the experimental data is presented. In section 3 we are presenting the evolution equations that describe the $Q^{2}$ dependence of polarised parton densities including polarised tensorgluons. In section 4 the solution of the evolution equations are considered in leading and next to leading order. In Appendix we present the polarised splitting functions including tensorgluons and the corresponding anomalous dimensions $[1,2]$. 


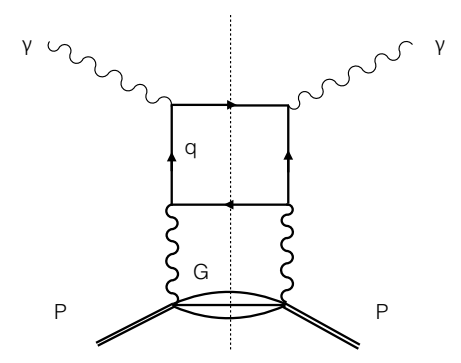

Figure 2. The typical diagram contributing to the scattering of a photon on a proton gluon $\mathrm{G}$ through the quark exchange.

\section{The Polarised Electroproduction}

The polarised electroproduction is described by two structure functions $G_{1}$ and $G_{2}$ in addition to the unpolarised structure functions $F_{1}$ and $F_{2}$. It is convenient to use the scaling functions $g_{1}\left(x, Q^{2}\right)=$ $M(p \cdot q) G_{1}$ and $g_{2}\left(x, Q^{2}\right)=(p \cdot q)^{2} G_{2} / M$, where $\mathrm{p}$ and $\mathrm{M}$ are the momentum and mass of the nucleon, $q$ is the momentum of the virtual photon with $Q^{2}=-q^{2}$ and $x=Q^{2} / 2(p \cdot q)$. These spin-dependent structure functions $g_{i}$ can be extracted from measurements where longitudinally polarised leptons are scattered from a target that is polarised either longitudinally or transversely relative to the electron beam [10]. For longitudinal beam and target polarisation, the difference between the cross sections for spins aligned and antialigned is dominated at high energy by the $g_{1}$ structure function. The $g_{2}$ structure function can be determined with additional measurement of cross sections for a nucleon polarised in a direction transverse to the beam polarisation. The moments of the spin structure functions are defined as

$$
\int_{0}^{1} d x x^{n-1} g_{i}\left(x, Q^{2}\right), \quad i=1,2 .
$$

In analogy with the unpolarised $F_{1}$ structure function

$$
F_{1}=\frac{1}{2} \sum_{i} e_{i}^{2}\left[q^{i}\left(x, Q^{2}\right)+\bar{q}^{i}\left(x, Q^{2}\right)\right]
$$

the structure function $g_{1}$ can be expressed at leading order of perturbation theory in terms of differences between quark distributions with spins aligned $q_{+}^{i}$ and antialigned $q_{-}^{i}$ relative to that of the nucleon $\Delta q^{i}=q_{+}^{i}-q_{-}^{i}$. Thus in zero order of the perturbative QCD, i.e. in the limit of the free quark-parton model the spin-dependent structure function $g_{1}$ can be expressed as [20,22]

$$
g_{1}=\frac{1}{2} \sum_{i} e_{i}^{2}\left[\Delta q^{i}\left(x, Q^{2}\right)+\Delta \bar{q}^{i}\left(x, Q^{2}\right)\right] .
$$

At large $Q^{2}$ the moments (3) are given by the OPE series with the expansion coefficients related to nucleon matrix elements of the twist-2 operators and Wilson coefficient functions in the form of QCD series in strong coupling constant $\alpha=g^{2} / 4 \pi$. The lowest $(n=1)$ moments of the spin-dependent 


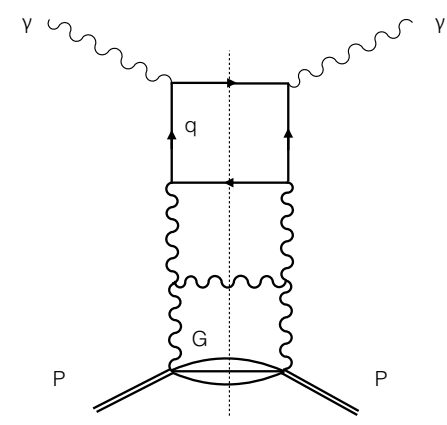

Figure 3. Example of the diagram demonstrating the scattering of a photon on a proton gluon $\mathrm{G}$ in the next to leading order through the quark and gluon exchange.

nucleon structure functions $g_{1}$ defined in (3) can be represented in the following form [17-20, 22, 30, 32]:

$$
\begin{aligned}
& \Gamma_{1}^{p}=\int_{0}^{1} d x g_{1}^{p}\left(x, Q^{2}\right)=I_{3}+I_{8}+I_{0} \\
& \Gamma_{1}^{n}=\int_{0}^{1} d x g_{1}^{n}\left(x, Q^{2}\right)=-I_{3}+I_{8}+I_{0},
\end{aligned}
$$

where

$$
\begin{aligned}
I_{3} & =\frac{1}{12} a_{3}\left(1-\frac{\alpha\left(Q^{2}\right)}{2 \pi}+\ldots\right) \\
I_{8} & =\frac{1}{36} a_{8}\left(1-\frac{\alpha\left(Q^{2}\right)}{2 \pi}+\ldots\right) \\
I_{0} & =\frac{1}{9} a_{0}\left(1-\frac{\alpha\left(Q^{2}\right)}{2 \pi} \frac{33-8 n_{f}}{33-2 n_{f}}+\ldots\right) .
\end{aligned}
$$

The matrix elements of the non-singlet axial currents $J_{5 \mu}^{k}$ and the singlet axial current $J_{5 \mu}$ are defined as follows ${ }^{1}$ :

$$
\begin{aligned}
<p, s\left|\bar{\psi} \gamma^{\mu} \gamma_{5} \frac{\lambda^{k}}{2} \psi\right| p, s> & =M s^{\mu} a_{k}, \quad k=1,2, \ldots, 8 \\
<p, s\left|\bar{\psi} \gamma^{\mu} \gamma_{5} \psi\right| p, s> & =2 M s^{\mu} a_{0} .
\end{aligned}
$$

The $\lambda^{k}$ are generators of the flavour group and $\psi=(u, d, s, \ldots)$ is a vector in flavour space. The $a_{k}, a_{0}$ are Lorentz invariant matrix elements and reflect the unknown, nonperturbative aspect of the QCD dynamics. The nucleon states $<p, s \mid$ are labeled by the momentum $p_{\mu}$ and the covariant spin vector $s_{\mu}\left(s \cdot p=0, s^{2}=-1\right)$. There are perturbative corrections to the coefficient functions which are included in expression (7).

To determine the flavour non-singlet components $a_{3}$ and $a_{8}$ on the right hand side of the equations (6), (7) one should turn to the beta-decay data [9, 10,30,31]. The values of $a_{3}$ and $a_{8}$ extracted from

\footnotetext{
${ }^{1}$ The quark distributions are defined in terms of SU(3)-flavour structure [9, 10]: NS hypercharge $a_{8}=\Delta u+\Delta d-$ $2 \Delta s, N S$ isovector $a_{3}=\Delta u-\Delta d$, S inglet $a_{0}=\Delta u+\Delta d+\Delta s$.
} 
hyperon beta-decays under the assumption of $\mathrm{SU}(3)$ flavour symmetry $\operatorname{are}^{2}[9,10,30,31,33]$ :

$$
\begin{array}{ll}
a_{8}=\Delta u+\Delta d-2 \Delta s=3 F-D & (0.58 \pm 0.03) \\
a_{3}=\Delta u-\Delta d=F+D & (1.270 \pm 0.003)
\end{array}
$$

The experimental value of the quarks spin density $\Delta \Sigma=a_{0}$ can be obtained through the measurements of the first moment integrals of $g_{1}(6) \Gamma_{1}^{p}=0.135 \pm 0.011$, (7) $\Gamma_{1}^{n}=-0.028 \pm 0.006$, of the strong coupling constant $\alpha_{s}=0.28 \pm 0.02$ all at $\left\langle Q^{2}\right\rangle=5 \mathrm{GeV}^{2}[30,34]$ and the knowledge of $a_{3}$ and $a_{8}$ in (9), thus

$$
a_{0}=\Delta \Sigma=\Delta u+\Delta d+\Delta s=0.33 \pm 0.03 .
$$

This value is unexpectedly small, compared with the naive expectation of $\Delta \Sigma_{S U(6)} \simeq 1$ and of the Ellis-Jaffe sum rule $\Delta \Sigma_{E J} \simeq 0.65$ [13]. By now it is experimentally well established that indeed the matrix element $a_{0}$ of the flavour-singlet axial-vector current is small and only of the order of $0.2-0.3$. The question raised was the following: why the quark spin content of the nucleon is so small?

One of the possible answers considered in the literature consists in the realisation that the singlet component $a_{0}$ receives an additional contribution from the gluon polarisation $\triangle G[20-23,35]$

$$
\Delta G=\int_{0}^{1} d x\left[G_{+}\left(x, Q^{2}\right)-G_{-}\left(x, Q^{2}\right)\right]
$$

which is the amount of spin carried by polarised gluons in the polarised nucleon.

In general, the value of the matrix elements (8) depend on the renormalisation scale $\mu^{2}$. Only if the operator is conserved, one can show that the matrix elements are $\mu^{2}$ independent [36]. Because the non-singlet currents $J_{5 \mu}^{3,8}$ are exactly conserved in the massless limit, the anomalous dimension of the non-singlet axial current vanishes at all orders. Thus $a_{3}$ and $a_{8}$ in (7) are renormalisation group invariant $\left(\mu^{2}\right.$ independent), they have a real physics meaning and are protected from substantial QCD radiative corrections [36]. Perturbative corrections to the coefficient functions are presented in (7) [17-23].

The singlet axial current $J_{5 \mu}=\bar{\psi} \gamma_{\mu} \gamma_{5} \psi$ in (8) is not conserved [27, 28]:

$$
\partial^{\mu} J_{5 \mu}=n_{f} \frac{\alpha}{2 \pi} \operatorname{tr} G \wedge G=n_{f} \frac{\alpha}{2 \pi} \partial^{\mu} K_{\mu} .
$$

Its expectation value $a_{0}\left(\mu^{2}\right)$ depends on the renormalisation point $\mu^{2}$ and it is therefore not a physical quantity and receives anomalous contribution [17-23]. Therefore the interpretation of the quark spindensity $a_{0}=\Delta \Sigma$ in (7), (8) and (10) as the matrix element of the flavour singlet axial charge was reconsidered [20-23]. The modified singlet axial current

$$
J_{5 \mu}-n_{f} \frac{\alpha}{2 \pi} K_{\mu}
$$

is conserved, as follows from (12), and its matrix element defines the invariant spin-density, which includes the polarisation of the gluons $\Delta G$ in the nucleon

$$
<p, s\left|J_{5 \mu}-n_{f} \frac{\alpha}{2 \pi} K_{\mu}\right| p, s>=2 M s^{\mu} \hat{a}_{0}
$$

where now [22]

$$
\hat{a}_{0}=\Delta \Sigma-n_{f} \frac{\alpha}{2 \pi} \Delta G=\Delta u+\Delta d+\Delta s-n_{f} \frac{\alpha}{2 \pi} \Delta G
$$

\footnotetext{
${ }^{2}$ The invariant matrix elements $F$ and $D$ measured in beta-decay are: $F=0.459 \pm 0.008$ and $D=0.798 \pm 0.008$. [9, 10].
} 


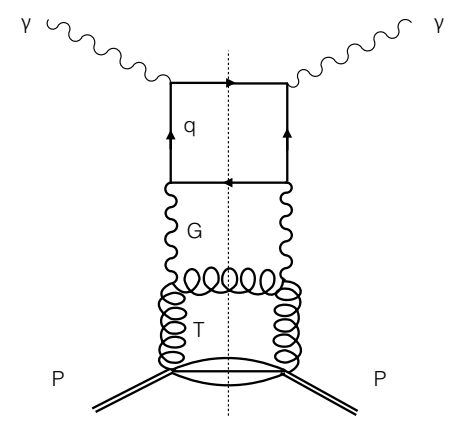

Figure 4. Example of the diagram demonstrating the scattering of a photon on a tensorgluon T, which appears in the next to leading order through the quark and gluon exchange.

Thus the matrix elements of the flavor singlet axial vector current are determined not solely by the first moment of the quark distributions, but also by the first moment of a spin-dependent gluon distribution $\Delta G$. This leads to possible explanations for the small value of $\hat{a}_{0}$ extracted from polarised deep inelastic experiments (10) that have been suggested in the literature [20, 22], which includes a spin screening (15) from positive gluon polarisation $\Delta G$. From the evolution equations it follows that the gluon polarisation grow as

$$
\Delta G\left(Q^{2}\right) \sim \frac{\text { const }}{\alpha\left(Q^{2}\right)}
$$

at large $Q^{2}$. This corresponds to a scaling contribution $\alpha\left(Q^{2}\right) \Delta G\left(Q^{2}\right)=$ const to the first moment $I_{e p, e n}(6)$ of $g_{1}$ (see formulas (41) and (42)). This suggestion sparked a program to measure $\Delta G$ which finally provides the first evidence for nonzero gluon polarisation in the proton $[6,12,15]$.

The polarised gluon distribution function $\Delta G$ was obtained from a QCD fit to the data on the spindependent structure function $g_{1}$. The QCD fits for $\Delta G$ were found in $[6,12,15]$, one with a positive and one with a negative first moment $\Delta G$, which describe the data equally well. The absolute value is of the order of $|\Delta G| \simeq 0.2-0.3$ for $Q^{2}=3 G e V^{2}$ and the uncertainty from the fit is of the order of 0.1 . This amount of gluon polarisation is not, by itself, sufficient to resolve the problem of the small value of $\hat{a}_{0}$. However that gluon polarisation $\Delta G \approx 0.2-0.3$ would still make a significant contribution to the nucleon polarisation $[6,12,15,16]$.

Considering the influence of polarised tensorgluons density $\Delta T_{s}$ to the spin of the nucleon we found that $I_{0}=I_{0}^{\prime}+I_{0}^{\prime \prime}$, the singlet component of the first moments of $g_{1}$ in (6) receives additional contribution $I_{0}^{\prime \prime}$ from tensorgluons (see Fig. 4):

$$
\begin{aligned}
& I_{0}^{\prime}=\frac{1}{9}\left(\Delta \Sigma-n_{f} \frac{\alpha\left(Q^{2}\right)}{2 \pi} \Delta G\right)\left(1-\frac{\alpha\left(Q^{2}\right)}{2 \pi} \frac{3\left(12 s^{2}-1\right)-8 n_{f}}{3\left(12 s^{2}-1\right)-2 n_{f}}+\ldots\right), \\
& I_{0}^{\prime \prime}=n_{f} \frac{\alpha\left(Q^{2}\right)}{2 \pi} \Delta T_{s} \frac{\sum_{k=1}^{2 s+1} \frac{1}{k}}{3\left(12 s^{2}-1\right)-2 n_{f}}+\ldots
\end{aligned}
$$

In the absence of tensorgluons ( $\mathrm{s}=1)$ the $I_{0}^{\prime}$ in (16) reduces to the standard QCD result (7) and $I_{0}^{\prime \prime}=0$ $(\Delta T=0)$. In the presence of the tensorgluons of spin $\mathrm{s}$ the result depends on the spin factor $\left(12 s^{2}-1\right)$, which appears in the Callan-Simanzik beta function coefficient $[1,2]$, as well as on the contribution 
of the tensorgluon density $\Delta T_{s}$, which scales similar to the quark density $\Delta \Sigma$ (see formulas (36) and (37)). In general, one should sum there over all "active" tensorgluon spins. It is difficult to assist the effect of the tensorgluons because it is not known if $\Delta T_{s}$ is positive or negative. Note that if $\Delta T_{s}$ is negative, i.e. if tensorgluons give negative contribution to the proton helicity, then the sign is correct for additional compensation of $\Delta \Sigma$ to occur.

In the next sections we shall derive evolution equations which include the tensorgluon polarisation and their contribution to the singlet part of the structure functions.

\section{Polarisation of Tensorgluons in Nucleon}

Our aim is to analyse a possible contribution of polarised tensorgluons $\Delta T_{s}$ into the spin of the nucleon if they are present in a nucleon. For that one should generalise the evolution equations for quark, gluons and tensorgluons derived in $[1,2]$ into the case of polarised tensorgluons. The generalisation of the DGLAP evolution equations which account for polarisation of tensorgluons can be written in the following form:

$$
\begin{aligned}
& \frac{d q_{+}^{i}(x, t)}{d t}=\frac{\alpha(t)}{2 \pi} \int_{x}^{1} \frac{d y}{y}\left[q_{+}^{i}(y, t) P_{q_{+} q_{+}}\left(\frac{x}{y}\right)+q_{-}^{i}(y, t) P_{q_{+} q_{-}}\left(\frac{x}{y}\right)+\right. \\
& \left.+G_{+}(y, t) P_{q_{+} G_{+}}\left(\frac{x}{y}\right)+G_{-}(y, t) P_{q_{+} G_{-}}\left(\frac{x}{y}\right)\right] \\
& \frac{d q_{-}^{i}(x, t)}{d t}=\frac{\alpha(t)}{2 \pi} \int_{x}^{1} \frac{d y}{y}\left[q_{+}^{i}(y, t) P_{q_{-} q_{+}}\left(\frac{x}{y}\right)+q_{-}^{i}(y, t) P_{q_{-} q_{-}}\left(\frac{x}{y}\right)+\right. \\
& \left.+G_{+}(y, t) P_{q_{-} G_{+}}\left(\frac{x}{y}\right)+G_{-}(y, t) P_{q_{-} G_{-}}\left(\frac{x}{y}\right)\right] \\
& \frac{d G_{+}(x, t)}{d t}=\frac{\alpha(t)}{2 \pi} \int_{x}^{1} \frac{d y}{y}\left[\sum_{i=1}^{2 f} q_{+}^{i}(y, t) P_{G_{+} q_{+}}\left(\frac{x}{y}\right)+\sum_{i=1}^{2 f} q_{-}^{i}(y, t) P_{G_{+} q_{-}}\left(\frac{x}{y}\right)+\right. \\
& +G_{+}(y, t) P_{G_{+} G_{+}}\left(\frac{x}{y}\right)+G_{-}(y, t) P_{G_{+} G_{-}}\left(\frac{x}{y}\right)+ \\
& \left.+T_{+}(y, t) P_{G_{+} T_{+}}\left(\frac{x}{y}\right)+T_{-}(y, t) P_{G_{+} T_{-}}\left(\frac{x}{y}\right)\right] \\
& \frac{d G_{-}(x, t)}{d t}=\frac{\alpha(t)}{2 \pi} \int_{x}^{1} \frac{d y}{y}\left[\sum_{i=1}^{2 f} q_{+}^{i}(y, t) P_{G_{-} q_{+}}\left(\frac{x}{y}\right)+\sum_{i=1}^{2 f} q_{-}^{i}(y, t) P_{G_{-} q_{-}}\left(\frac{x}{y}\right)+\right. \\
& +G_{+}(y, t) P_{G_{-} G_{+}}\left(\frac{x}{y}\right)+G_{-}(y, t) P_{G_{-} G_{-}}\left(\frac{x}{y}\right)+ \\
& \left.+T_{+}(y, t) P_{G_{-} T_{+}}\left(\frac{x}{y}\right)+T_{-}(y, t) P_{G_{-} T_{-}}\left(\frac{x}{y}\right)\right] \\
& \frac{d T_{+}(x, t)}{d t}=\frac{a(t)}{2 \pi} \int_{x}^{1} \frac{d y}{y}\left[G_{+}(y, t) P_{T_{+} G_{+}}\left(\frac{x}{y}\right)+G_{-}(y, t) P_{T_{+} G_{-}}\left(\frac{x}{y}\right)+\right. \\
& \left.+T_{+}(y, t) P_{T_{+} T_{+}}\left(\frac{x}{y}\right)+T_{-}(y, t) P_{T_{+} T_{-}}\left(\frac{x}{y}\right)\right] \\
& \frac{d T_{-}(x, t)}{d t}=\frac{\alpha(t)}{2 \pi} \int_{x}^{1} \frac{d y}{y}\left[G_{+}(y, t) P_{T_{-} G_{+}}\left(\frac{x}{y}\right)+G_{-}(y, t) P_{T_{-} G_{-}}\left(\frac{x}{y}\right)+\right. \\
& +T_{+}(y, t) P_{T_{-} T_{+}}\left(\frac{x}{y}\right)+T_{-}(y, t) P_{T_{-} T_{-}}\left(\frac{x}{y}\right),
\end{aligned}
$$


where $t=\ln \left(Q^{2} / Q_{0}^{2}\right)$. This set of equations can be simplified by observing that parity conservation in QCD implies the relations

$$
P_{B_{+} A_{ \pm}}(z)=P_{B_{-} A_{\mp}}(z)
$$

for any $\mathrm{A}$ and $\mathrm{B}$ and that the sums and the differences

$$
\begin{gathered}
q^{i}=q_{+}^{i}+q_{-}^{i}, \quad G=G_{+}+G_{-}, \quad T=T_{+}+T_{-}, \\
\Delta q^{i}=q_{+}^{i}-q_{-}^{i}, \quad \Delta G=G_{+}-G_{-}, \quad \Delta T=T_{+}-T_{-}
\end{gathered}
$$

evolve separately. Physically the quantities $\Delta q^{i}, \Delta G, \Delta T$ represent the differences between the number densities of quarks, gluons and tensorgluons with spin parallel to the nucleon spin and those with spin anti-parallel. It is also convenient to define the sum and the difference of splitting functions:

$$
P_{B A}(z)=P_{B_{+} A_{+}}(z)+P_{B_{-} A_{+}}(z), \quad \Delta P_{B A}(z)=P_{B_{+} A_{+}}(z)-P_{B_{-} A_{+}}(z) .
$$

We derive then from (18) the master equation for differences:

$$
\begin{gathered}
\frac{d}{d t} \Delta q^{i}(x, t)=\frac{\alpha(t)}{2 \pi} \int_{x}^{1} \frac{d y}{y}\left[\Delta q^{i}(y, t) \Delta P_{q q}\left(\frac{x}{y}\right)+\Delta G(y, t) \Delta P_{q G}\left(\frac{x}{y}\right)\right] \\
\frac{d}{d t} \Delta G(x, t)=\frac{\alpha(t)}{2 \pi} \int_{x}^{1} \frac{d y}{y}\left[\sum_{i=1}^{2 n_{f}} \Delta q^{i}(y, t) \Delta P_{G q}\left(\frac{x}{y}\right)+\Delta G(y, t) \Delta P_{G G}\left(\frac{x}{y}\right)+\Delta T(y, t) \Delta P_{G T}\left(\frac{x}{y}\right)\right] \\
\frac{d}{d t} \Delta T(x, t)=\frac{\alpha(t)}{2 \pi} \int_{x}^{1} \frac{d y}{r y}\left[\Delta G(y, t) \Delta P_{T G}\left(\frac{x}{y}\right)+\Delta T(y, t) \Delta P_{T T}\left(\frac{x}{y}\right)\right] .
\end{gathered}
$$

In the case of non-singlet (NS) quark densities the above equation reduces to the one of the standard QCD:

$$
\frac{d}{d t} \Delta q^{N S}(x, t)=\frac{\alpha(t)}{2 \pi} \int_{x}^{1} \frac{d y}{y} \Delta q^{N S}(y, t) \Delta P_{q q}\left(\frac{x}{y}\right),
$$

where $q^{N S}(y, t)=q(x, t)-\bar{q}(x, t)$. The splitting function $P_{q_{-} q_{+}}=0$ and therefore $\Delta P_{q q}=P_{q_{+} q_{+}}=P_{q q}$. It follows then that the moments of $\Delta q^{N S}$ evolve in $Q^{2}$ with the same logarithmic exponent as $q^{N S}$ and therefore as the non-singlet parts of $F_{1}$ and $g_{1}$.

The integral

$$
\Delta q^{i}=\int_{0}^{1} d x\left[\Delta q^{i}(x, t)+\Delta \bar{q}^{i}(x, t)\right]
$$

represents the fraction of the proton's spin carried by the quarks and the anti-quarks of the flavour $q^{i}$. The summation over all quark flavour contributions gives the total fraction of the nucleon spin carried by light quark and antiquarks (below the contribution of the heavy quarks has been neglected):

$$
\Delta \Sigma=\int_{0}^{1} d x \sum_{i}\left[\Delta q^{i}(x, t)+\Delta \bar{q}^{i}(x, t)\right]=\Delta u+\Delta d+\Delta s .
$$

As we already reviewed in the introduction, the conservation of the SU(3) singlet axial current, valid for massless quarks, is broken in QCD by the Adler-Bell-Jackiw anomaly [27, 28]. While the anomalous dimension of the non-singlet axial current vanishes at all orders [17, 18], the singlet axial current anomalous dimension is different from zero. Due to this non-conservation in the singlet sector there is a gluon contribution at all values of $Q^{2}$ in the singlet part of the integral of $g_{1}$. This gluon term $\Delta G$ in (15)

$$
\hat{a}_{0}=\Delta \Sigma-n_{f} \frac{\alpha}{2 \pi} \Delta G
$$




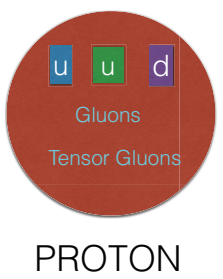

Figure 5. We assume that inside the hadrons there are additional partons - tensorgluons - which carry the same charges as the gluons, but have larger spin $[1,2]$. The nucleon's spin is accounted then by the quark spin $\frac{1}{2} \Delta \Sigma$, gluon spin $1 \Delta G$, as well as by the tensorgluon spin $s \Delta T_{s}$ and the total orbital angular momentum $L_{z}(28)$.

can in principle be large and we are interested to consider a possible contribution of the tensorgluons polarisation as well. With this aim we shall introduce in addition to the gluon polarisation the corresponding tensorgluon helicity content in the polarised nucleon:

$$
\Delta G=\int_{0}^{1} d x\left[G_{+}\left(x, Q^{2}\right)-G_{-}\left(x, Q^{2}\right)\right], \quad \Delta T=\int_{0}^{1} d x\left[T_{+}\left(x, Q^{2}\right)-T_{-}\left(x, Q^{2}\right)\right],
$$

with $G_{ \pm}, T_{ \pm}$being the gluon and tensorgluons densities with helicities \pm 1 and $\pm s$ respectively in a nucleon with helicity $+\frac{1}{2} . \Delta G$ represents the amount of helicity carried by the gluons in the nucleon and $\Delta T_{s}$, the amount carried by the tensorgluons.

The nucleon's spin is accounted by the quarks' spin as well as by gluon and tensorgluon spins $\Delta G$, $\Delta T_{s}$ and the total orbital angular momentum $L_{z}$. Because the spin of the nucleon is $1 / 2$ one should have

$$
\frac{1}{2} \Delta \Sigma+\Delta G+\sum_{s} s \Delta T_{s}+L_{z}=\frac{1}{2}
$$

In this equation the helicity of the gluon is equal to one and of tensorgluon is $s=2,3, .$. , and the summation is over all "active" tensor bosons in the nucleon. The various components of the nucleon spin in the above equation can be measured experimentally. The gluon sector $\Delta G$ can be measured in deep inelastic scattering experiments in which gluon-gluon or gluon-quark scattering dominates. One of the most interesting suggestions to come out of the spin crisis is that $\Delta G$ may contribute significantly to the nucleon. Our aim is to consider a possible contribution of the tensorgluon spin $s \triangle T_{s}$.

\section{Evolution Equation in Leading and Next to Leading Order}

At leading order the QCD evolution of $\Delta \Sigma, \Delta G$ and $\Delta T$ can written from (22) as

$$
\frac{d}{d t}\left(\begin{array}{c}
\Delta \Sigma \\
\Delta G \\
\Delta T
\end{array}\right)=\frac{\alpha(t)}{2 \pi}\left(\begin{array}{ccc}
\Delta \gamma_{q q}^{(1)} & 2 n_{f} \Delta \gamma_{q G}^{(1)} & 0 \\
\Delta \gamma_{G q}^{(1)} & \Delta \gamma_{G G}^{(1)} & \Delta \gamma_{G T}^{(1)} \\
0 & \Delta \gamma_{T G}^{(1)} & \Delta \gamma_{T T}^{(1)}
\end{array}\right)\left(\begin{array}{c}
\Delta \Sigma \\
\Delta G \\
\Delta T
\end{array}\right),
$$


where [2] (see Appendix A for the values of the corresponding anomalous dimensions)

$$
\Delta \gamma_{1}=\left(\begin{array}{ccc}
0 & 0 & 0 \\
\frac{3}{2} C_{2}(R) & C_{2}(G) \frac{\sum_{s}\left(12 s^{2}-1\right)}{6}-\frac{2}{3} n_{f} T(R) & C_{2}(G) \sum_{k=1}^{2 s+1} \frac{1}{k} \\
0 & 0 & 0
\end{array}\right),
$$

and $C_{2}(G)=N, C_{2}(R)=\frac{N^{2}-1}{2 N}, T(R)=\frac{1}{2}$ for the SU(N) groups. Let us represent the $\Delta \gamma_{1}$ as

$$
\Delta \gamma_{1}=\left(\begin{array}{ccc}
0 & 0 & 0 \\
C_{1} & b & C_{3} \\
0 & 0 & 0
\end{array}\right)
$$

where [2]

$$
C_{1}=\frac{3}{2} C_{2}(R), \quad b=C_{2}(G) \frac{\sum_{s}\left(12 s^{2}-1\right)}{6}-\frac{2}{3} n_{f} T(R), \quad C_{3}=C_{2}(G) \sum_{k=1}^{2 s+1} \frac{1}{k}
$$

and

$$
\frac{d \alpha(t)}{d t}=-\frac{b}{2 \pi} \alpha^{2}(t)
$$

The eigenvalues of the matrix $\Delta \gamma_{1}$ are $\lambda_{1}=b, \lambda_{2,3}=0$ and the corresponding eigenvectors

$$
S=\left(\begin{array}{ccc}
-\frac{C_{3}}{C_{1}} & -\frac{b}{C_{1}} & 0 \\
0 & 1 & 1 \\
1 & 0 & 0
\end{array}\right), \quad S^{-1}=\left(\begin{array}{ccc}
0 & 0 & 1 \\
-\frac{C_{1}}{b} & 0 & -\frac{C_{3}}{b} \\
\frac{C_{1}}{b} & 1 & \frac{C_{3}}{b}
\end{array}\right)
$$

define the transformation $\Delta \gamma_{1}=S \Lambda S^{-1}$, where $\Lambda=\operatorname{diag}(0,0, b)$, which allows to represent the evolution equation (29) in the following form:

$$
\frac{d}{d t}\left(\begin{array}{c}
\Delta \Sigma \\
\Delta G \\
\Delta T
\end{array}\right)=\frac{\alpha(t)}{2 \pi} S \Lambda S^{-1}\left(\begin{array}{c}
\Delta \Sigma \\
\Delta G \\
\Delta T
\end{array}\right)
$$

Multiplying this equation from the left by $S^{-1}$ we shall get

$$
\frac{d}{d t}\left[S^{-1}\left(\begin{array}{c}
\Delta \Sigma \\
\Delta G \\
\Delta T
\end{array}\right)\right]=\frac{\alpha(t)}{2 \pi} \Lambda\left[S^{-1}\left(\begin{array}{c}
\Delta \Sigma \\
\Delta G \\
\Delta T
\end{array}\right)\right]
$$

and it is useful to introduce the new coordinates

$$
\left(\begin{array}{l}
y_{1}(t) \\
y_{2}(t) \\
y_{3}(t)
\end{array}\right)=S^{-1}\left(\begin{array}{c}
\Delta \Sigma \\
\Delta G \\
\Delta T
\end{array}\right)=\left(\begin{array}{c}
\Delta T \\
-\frac{1}{b}\left(C_{1} \Sigma+C_{3} \Delta T\right) \\
\frac{C_{1}}{b} \Sigma+\Delta G+\frac{C_{3}}{b} \Delta T
\end{array}\right) .
$$

The evolution equation takes a diagonal form:

$$
\frac{d}{d t}\left(\begin{array}{l}
y_{1}(t) \\
y_{2}(t) \\
y_{3}(t)
\end{array}\right)=\frac{\alpha(t)}{2 \pi}\left(\begin{array}{lll}
0 & 0 & 0 \\
0 & 0 & 0 \\
0 & 0 & b
\end{array}\right)\left(\begin{array}{l}
y_{1}(t) \\
y_{2}(t) \\
y_{3}(t)
\end{array}\right) .
$$

The first equation gives $\frac{d y_{1}(t)}{d t}=\frac{d \Delta T(t)}{d t}=0$, and we conclude that the helicity density of tensorgluons does not evolve with $Q^{2}$ :

$$
\Delta T(t)=\Delta T
$$


From this and from the second equation $d y_{2}(t) / d t=0$ it follows that the quark helicity distribution also does not evolve with $Q^{2}(d \Delta \Sigma(t) / d t=0)$ :

$$
\Delta \Sigma(t)=\Delta \Sigma .
$$

From the last equation $\frac{d y_{3}(t)}{d t}=\frac{\alpha(t)}{2 \pi} b y_{3}(t)$ it follows then that

$$
\frac{d \alpha(t) y_{3}(t)}{d t}=\frac{d \alpha(t)}{d t} y_{3}(t)+\alpha(t) \frac{d y_{3}(t)}{d t}=-\frac{b}{2 \pi} \alpha^{2}(t) y_{3}(t)+\alpha(t) \frac{\alpha(t)}{2 \pi} b y_{3}(t)=0,
$$

where we use (32). Thus

$$
\alpha(t) y_{3}(t)=\text { Const }
$$

and therefore from the last equation in (34)

$$
\frac{\alpha(t)}{2 \pi} \Delta G(t)=\text { Const }-\frac{\alpha(t)}{2 \pi b}\left[C_{3} \Delta T+C_{1} \Delta \Sigma\right] \rightarrow \text { Const } .
$$

as $t \rightarrow \infty$ and $\alpha(t) \rightarrow 0$. Here we use the fact that $\Delta \Sigma$ and $\Delta T$ are t-independent (36), (37). In the absence of tensorgluons the equation reduces to the Altarelli-Ross equation:

$$
\frac{\alpha(t)}{2 \pi} \Delta G(t)=\text { Const }-\frac{\alpha(t)}{2 \pi b} C_{1} \Delta \Sigma \rightarrow \text { Const. }
$$

Therefore it is natural to introduce the quantity [22]

$$
\Delta \Gamma=\frac{\alpha(t)}{2 \pi} \Delta G(t)
$$

and consider its behaviour in the next to leading order. One should notice that the coefficient in front of the tensorgluon density $C_{3}$ in (40) grows with spin as $\ln s$, therefore its contribution can still be numerically large for higher spins. The main conclusion which follows from the above consideration is that the densities $\Delta \Sigma$ and $\Delta T$ are $Q^{2}$ independent and that the gluon density grows with $Q^{2}$ as $\Delta G \approx 1 / \alpha\left(Q^{2}\right)$.

Expanding the splitting functions and anomalous dimensions into perturbative series

$$
\Delta P_{i j}=\Delta P_{i j}^{(1)}+\frac{a_{s}(t)}{2 \pi} \Delta P_{i j}^{(2)}+\left(\frac{a_{s}(t)}{2 \pi}\right)^{2} \Delta P_{i j}^{(3)}+\ldots
$$

one can get from (22):

$$
\frac{d}{d t}\left(\begin{array}{c}
\Delta \Sigma(t) \\
\Delta \Gamma(t) \\
\Delta T(t)
\end{array}\right)=\left(\frac{a_{s}(t)}{2 \pi}\right)^{2}\left(\begin{array}{ccc}
\Delta \gamma_{q q}^{(2)} & 2 n_{f} \Delta \gamma_{q G}^{(3)} & 0 \\
\Delta \gamma_{G q}^{(1)} & \Delta \gamma_{G G}^{(2)} & \Delta \gamma_{G T}^{(1)} \\
0 & \Delta \gamma_{T G}^{(3)} & \Delta \gamma_{T T}^{(2)}
\end{array}\right)\left(\begin{array}{c}
\Delta \Sigma(t) \\
\Delta \Gamma(t) \\
\Delta T(t)
\end{array}\right) .
$$

Here we have taken into account that there are no direct interactions between tensorgluons and quarks $\gamma_{T q}^{(2)}=\gamma_{q T}^{(2)}=0[1,2]$. The $Q^{2}$ evolution of the singlet component of the first moment (6) is given by the short-distance operator product expansion [17-19, 22]:

$$
I_{0}=\frac{1}{9} c\left(a_{s}(t)\right) E\left(a_{s}, a_{s}(t)\right) O\left(a_{s}\right),
$$

where we take into account the presence of the tensorgluons:

$$
c\left(a_{s}(t)\right)=\left(1+c_{\Sigma} a_{s}(t), \quad c_{\Gamma}+c_{\Gamma}^{\prime} a_{s}(t), \quad c_{T}+c_{T}^{\prime} a_{s}(t)\right), \quad O\left(a_{s}\right)=\left(\begin{array}{c}
\Delta \Sigma(t) \\
\Delta \Gamma(t) \\
\Delta T(t)
\end{array}\right)
$$


and

$$
E\left(a_{s}, a_{s}(t)\right)=1+\frac{a_{s}-a_{s}(t)}{4 \pi b} \Delta \gamma,
$$

$\gamma$ is the matrix of anomalous dimensions in equation (44). Substituting the matrix of anomalous dimensions (47), (44) and the vectors (46) into the (45) we shall get

$$
\begin{aligned}
I_{0}=\frac{1}{9} & \left\{\left[1+\frac{a_{s}-a_{s}(t)}{4 \pi b}\left(\Delta \gamma_{q q}^{(2)}+c_{\Gamma} \Delta \gamma_{G q}^{(1)}-b c_{\Sigma}\right)+c_{\Sigma} a_{s}\right] \Delta \Sigma+\right. \\
+ & {\left[1+\frac{a_{s}-a_{s}(t)}{4 \pi b}\left(\Delta \gamma_{G G}^{(2)}+\frac{1}{c_{\Gamma}} \Delta \gamma_{q G}^{(3)}+\frac{c_{T}}{c_{\Gamma}} \Delta \gamma_{T G}^{(3)}-b \frac{c_{\Gamma}^{\prime}}{c_{\Gamma}}\right)+\frac{c_{\Gamma}^{\prime}}{c_{\Gamma}} a_{s}\right] c_{\Gamma} \Delta \Gamma+} \\
+ & {\left.\left[c_{T}+\frac{a_{s}-a_{s}(t)}{4 \pi b}\left(c_{T} \Delta \gamma_{T T}^{(2)}+c_{\Gamma} \Delta \gamma_{G T}^{(1)}-b c_{T}^{\prime}\right)+c_{T}^{\prime} a_{s}\right] \Delta T\right\} . }
\end{aligned}
$$

One can see the appearance of the tensorgluon contribution $\Delta T$ into the first moment of the singlet polarised structure function. In this expression the anomalous dimensions associated with the quark and gluon interactions were computed in [17-19,22]. These values are $\Delta \gamma_{q q}^{(2)}=0$ and

$$
\begin{aligned}
c_{\Gamma} & =-n_{f}, \quad c_{\Sigma}=-\frac{3}{4 \pi} C_{2}(R), \quad c_{\Gamma}^{\prime}=c_{\Gamma} c_{\Sigma} \\
\Delta \gamma_{G q}^{(1)} & =\frac{3}{2} C_{2}(R), \quad \Delta \gamma_{q G}^{(3)}=c_{\Gamma}^{2} \Delta \gamma_{G q}^{(1)}, \Delta \gamma_{G G}^{(2)}=0 .
\end{aligned}
$$

Because there are no interactions between photon and tensorgluons through the quark exchanges, the coefficients $c_{T}$ and $c_{T}^{\prime}$ vanish. Hence, keeping terms proportional to $\alpha(t)$ and using the above values for the anomalous dimensions, the first moment of the singlet part of the proton structure function (48) takes the following form:

$$
\begin{aligned}
I_{0}^{\prime} & =\frac{1}{9}\left[1-\frac{a_{s}(t)}{4 \pi b}\left(c_{\Gamma} \Delta \gamma_{G q}^{(1)}-b c_{\Sigma}\right)\right]\left(\Delta \Sigma-n_{f} \Delta \Gamma\right) \\
I_{0}^{\prime \prime} & =-\frac{1}{9}\left[\frac{a_{s}(t)}{4 \pi b} c_{\Gamma} \Delta \gamma_{G T}^{(1)}\right] \Delta T,
\end{aligned}
$$

where $I_{0}=I_{0}^{\prime}+I_{0}^{\prime \prime}$. The coefficient of the beta function $b$ of the running coupling constant is given in (31). Given that $C_{2}(G)=3, C_{2}(R)=\frac{4}{3}$ and $T(R)=\frac{1}{2}$ we finally get:

$$
\begin{aligned}
& I_{0}^{\prime}=\frac{1}{9}\left(\Delta \Sigma-n_{f} \frac{\alpha_{s}(t)}{2 \pi} \Delta G\right)\left(1-\frac{a_{s}(t)}{2 \pi} \frac{3 \sum\left(12 s^{2}-1\right)-8 n_{f}}{3 \sum\left(12 s^{2}-1\right)-2 n_{f}}+\ldots\right) \\
& I_{0}^{\prime \prime}=n_{f} \sum_{s} \frac{a_{s}(t)}{2 \pi} \Delta T_{s} \frac{\sum_{k=1}^{2 s+1} \frac{1}{k}}{3 \sum\left(12 s^{2}-1\right)-2 n_{f}}+\ldots
\end{aligned}
$$

Comparing this result with the standard QCD case one can see that the assumed presence of the tensorgluons inside the proton modifies the singlet part of the polarised proton structure function in two ways. On the one hand, there is a contribution $3 \sum\left(12 s^{2}-1\right)$ in the numerator and the denominator to the radiative correction $I_{0}^{\prime}$ which effectively lowers its value. On the other hand, there is an additional contribution $I_{0}^{\prime \prime}$ of the tensorgluons $\Delta T_{s}$. The overall sign of this contribution depends on the values of the spin $s$ of the tensorgluon and of its density $\Delta T_{s}$. Note that if $\Delta T_{s}$ is negative, i.e. if tensorgluons give negative contribution to the proton helicity, then the sign is correct for the additional compensation of $\Delta \Sigma$ to occur. 


\section{Acknowledgements}

On of us (G.S.) would like to thank the organisers of the International Seminar on High Energy Physics "Quarks-2016" for invitation and kind hospitality.

\section{Appendix A}

Since $P_{B_{+} A_{ \pm}}(z)=P_{B_{-} A_{\mp}}(z)$, we only need to evaluate $P_{B_{+} A_{+}}(z), P_{B_{-} A_{+}}(z)$. Then

$$
P_{B A}(z)=P_{B_{+} A_{+}}(z)+P_{B_{-} A_{+}}(z), \quad \Delta P_{B A}(z)=P_{B_{+} A_{+}}(z)-P_{B_{-} A_{+}}(z) .
$$

Hence for the $\Delta P_{q q}[24]$ :

$$
\begin{aligned}
P_{q_{-} q_{+}}(z) & =0 \\
\Delta P_{q q}=P_{q_{+} q_{+}}(z)=P_{q q}= & =C_{2}(R)\left[\frac{1+z^{2}}{(1-z)_{+}}+\frac{3}{2} \delta(z-1)\right],
\end{aligned}
$$

for the $\Delta P_{G q}(z)$

$$
\begin{aligned}
P_{G_{+} q_{+}}(z)=C_{2}(R) \frac{1}{z}, & P_{G_{-} q_{+}}(z)=C_{2}(R) \frac{(1-z)^{2}}{z} \\
P_{G q}(z)=P_{G_{+} q_{+}}(z)+P_{G_{-} q_{+}}(z)= & C_{2}(R) \frac{1+(1-z)^{2}}{z} \\
\Delta P_{G q}(z)=P_{G_{+} q_{+}}(z)-P_{G_{-} q_{+}}(z)= & C_{2}(R) \frac{1-(1-z)^{2}}{z}
\end{aligned}
$$

for the $\triangle P_{q G}(z)$

$$
\begin{aligned}
P_{q_{+} G_{+}}(z)=\frac{z^{2}}{2}, & P_{q_{-} G_{+}}(z)=\frac{(z-1)^{2}}{2} \\
P_{q G}(z)=P_{q_{+} G_{+}}(z)+P_{q_{-} G_{+}}(z) & =\frac{z^{2}+(z-1)^{2}}{2} \\
\triangle P_{q G}(z)=P_{q_{+} G_{+}}(z)-P_{q_{-} G_{+}}(z) & =\frac{z^{2}-(z-1)^{2}}{2}
\end{aligned}
$$

as well as for the $\triangle P_{G G}(z)$

$$
\begin{aligned}
P_{G_{+} G_{+}}(z) & =C_{2}(G)\left[\left(1+z^{4}\right)\left(\frac{1}{z}+\frac{1}{(1-z)_{+}}\right)+\left(\frac{\sum_{s}\left(12 s^{2}-1\right)}{6}-\frac{2}{3} \frac{T(R)}{C_{2}(G)}\right) \delta(z-1)\right] \\
P_{G_{-} G_{+}}(z) & =C_{2}(G) \frac{(1-z)^{3}}{z} \\
\Delta P_{G G}(z) & =C_{2}(G)\left[\left(1+z^{4}\right)\left(\frac{1}{z}+\frac{1}{(1-z)_{+}}\right)-\frac{(1-z)^{3}}{z}+\left(\frac{\sum_{s}\left(12 s^{2}-1\right)}{6}-\frac{2}{3} \frac{T(R)}{C_{2}(G)}\right) \delta(z-1)\right] .
\end{aligned}
$$

The behaviour at small $x \rightarrow 0$ of the unpolarised and polarised splitting functions is different

$$
\lim _{x \rightarrow 0} P_{G G}(z)=C_{2}(G)\left(\frac{1}{z}+\frac{1}{z}\right), \quad \lim _{x \rightarrow 0} \Delta P_{G G}(z)=C_{2}(G)\left(\frac{1}{z}-\frac{1}{z}\right)=0 .
$$


The polarised splitting function $\Delta P_{G G}(z)$ has moderate behaviour at small x's. The polarised splitting functions which are including tensorgluons were found in $[1,2]$. For the non-diagonal functions they are:

$$
\begin{aligned}
P_{T_{+} G_{+}}(z) & =C_{2}(G) \frac{z^{2 s+1}}{(1-z)^{2 s-1}} \\
P_{T_{-} G_{+}}(z) & =C_{2}(G) \frac{(1-z)^{2 s+1}}{z^{2 s-1}} \\
\Delta P_{T G}(z) & =C_{2}(G)\left[\frac{z^{2 s+1}}{(1-z)^{2 s-1}}-\frac{(1-z)^{2 s+1}}{z^{2 s-1}}\right]
\end{aligned}
$$

and at small $\mathrm{x}$ both functions are singular

$$
\lim _{x \rightarrow 0} P_{T G}(z)=C_{2}(G) \frac{1}{z^{2 s-1}}, \quad \lim _{x \rightarrow 0} \Delta P_{T G}(z)=-C_{2}(G) \frac{1}{z^{2 s-1}} .
$$

Comparing this behaviour with the gluon splitting functions (55) one can see that here the polarised splitting function has enhanced singular behaviour in the small x region. Finally for the $P_{T G}$ function we have

$$
\begin{aligned}
P_{G_{+} T_{+}}(z) & =C_{2}(G) \frac{1}{z(1-z)^{2 s-1}} \\
P_{G_{-} T_{+}}(z) & =C_{2}(G) \frac{(1-z)^{2 s+1}}{z} \\
\triangle P_{G T}(z) & =C_{2}(G)\left[\frac{1}{z(1-z)^{2 s-1}}-\frac{(1-z)^{2 s+1}}{z}\right] .
\end{aligned}
$$

In the small $\mathrm{x}$ region we have behaviour which is similar to gluon (55)

$$
\lim _{x \rightarrow 0} P_{G T}(z)=C_{2}(G) \frac{2}{z}, \quad \lim _{x \rightarrow 0} \Delta P_{G T}(z)=0
$$

The tensor-tensor splitting function is helicity conserving:

$$
\begin{aligned}
P_{T_{-} T_{+}}(z) & =0 \\
\Delta P_{T T}(z)=P_{T_{+} T_{+}}(z) & =C_{2}(G)\left[\frac{z^{2 s+1}}{(1-z)_{+}}+\frac{1}{(1-z) z_{+}^{2 s-1}}+\sum_{j=1}^{2 s+1} \frac{1}{j} \delta(z-1)\right]
\end{aligned}
$$

and is singular in the small $\mathrm{x}$ region both functions are singular

$$
\lim _{x \rightarrow 0} P_{T T}(z)=C_{2}(G) \frac{1}{z^{2 s-1}}, \quad \lim _{x \rightarrow 0} \Delta P_{T T}(z)=C_{2}(G) \frac{1}{z^{2 s-1}} .
$$

The matrix of anomalous dimensions is [24]:

$$
\int_{0}^{1} d z z^{n-1}\left(\begin{array}{ccc}
\Delta P_{q q}(z) & 2 n_{f} \Delta P_{q G}(z) & 0 \\
\Delta P_{G q}(z) & \Delta P_{G G}(z) & P_{G T}(z) \\
0 & \Delta P_{T G}(z) & \Delta P_{T T}(z)
\end{array}\right)=\left(\begin{array}{ccc}
\Delta \gamma_{q q}(n) & 2 n_{f} \Delta \gamma_{q G}(n) & 0 \\
\Delta \gamma_{G q}(n) & \Delta \gamma_{G G}(n) & \Delta \gamma_{G T}(n) \\
0 & \Delta \gamma_{T G}(n) & \Delta \gamma_{T T}(n)
\end{array}\right),
$$


where

$$
\begin{aligned}
\int_{0}^{1} d z \Delta P_{q q}(z) & =0, \quad n=1, \\
\int_{0}^{1} d z \Delta P_{q G}(z) & =0, \\
\int_{0}^{1} d z \Delta P_{G q}(z) & =\frac{3}{2} C_{2}(R), \\
\int_{0}^{1} d z \Delta P_{G G}(z) & =\frac{\sum_{s}\left(12 s^{2}-1\right)}{6} C_{2}(G)-\frac{2}{3} T(R),
\end{aligned}
$$

and for the tensorgluons $[1,2]$

$$
\begin{aligned}
& \int_{0}^{1} d z \Delta P_{G T}(z)=C_{2}(G) \sum_{k=1}^{2 s+1} \frac{1}{k}, \quad n=1, \\
& \int_{0}^{1} d z \Delta P_{T G}(z)=0, \\
& \int_{0}^{1} d z \Delta P_{T T}(z)=0 .
\end{aligned}
$$

\section{References}

[1] G. Savvidy, Asymptotic freedom of non-Abelian tensor gauge fields, Phys. Lett. B 732 (2014) 150.

[2] G. Savvidy, Proton structure and tensor gluons, J. Phys. A 47 (2014) 35, 355401 [arXiv:1310.0856 [hep-th]].

[3] J. Ashman et al. [European Muon Collaboration], An Investigation of the Spin Structure of the Proton in Deep Inelastic Scattering of Polarized Muons on Polarized Protons, Nucl. Phys. B 328 (1989) 1.

[4] P. L. Anthony et al. [E155 Collaboration], Measurements of the $Q^{2}$ dependence of the proton and neutron spin structure functions $g_{1}^{p}$ and $g_{1}^{n}$, Phys. Lett. B 493 (2000) 19.

[5] M. G. Alekseev et al. [COMPASS Collaboration.], The Spin-dependent Structure Function of the Proton $g_{1}^{p}$ and a Test of the Bjorken Sum Rule, Phys. Lett. B 690 (2010) 466.

[6] C. Adolph et al. [COMPASS Collaboration], Leading and Next-to-Leading Order Gluon Polarization in the Nucleon and Longitudinal Double Spin Asymmetries from Open Charm Muoproduction, Phys. Rev. D 87 (2013) 5, 052018 [arXiv:1211.6849 [hep-ex]].

[7] A. Airapetian et al. [HERMES Collaboration], Precise determination of the spin structure function g(1) of the proton, deuteron and neutron, Phys. Rev. D 75 (2007) 012007.

[8] D. de Florian, R. Sassot, M. Stratmann and W. Vogelsang, Extraction of Spin-Dependent Parton Densities and Their Uncertainties, Phys. Rev. D 80 (2009) 034030.

[9] M. Anselmino, A. Efremov and E. Leader, The Theory and phenomenology of polarised deep inelastic scattering, Phys. Rept. 261 (1995) 1 [Erratum-ibid. 281 (1997) 399] .

[10] C. A. Aidala, S. D. Bass, D. Hasch and G. K. Mallot, The Spin Structure of the Nucleon, Rev. Mod. Phys. 85 (2013) 655 [arXiv:1209.2803 [hep-ph]].

[11] P. Jimenez-Delgado, W. Melnitchouk and J. F. Owens, Parton momentum and helicity distributions in the nucleon, J. Phys. G 40 (2013) 093102 [arXiv:1306.6515 [hep-ph]].

[12] G. K. Mallot, Measurements of Delta G, AIP Conf. Proc. 915 (2007) 325 [hep-ex/0612055]. 
[13] J. R. Ellis and R. L. Jaffe, A Sum Rule for Deep Inelastic Electroproduction from Polarized Protons, Phys. Rev. D 9 (1974) 1444 [Erratum-ibid. D 10 (1974) 1669].

[14] J. R. Ellis and M. Karliner, Direct estimate of the gluon polarization in the nucleon, Mod. Phys. Lett. A 21 (2006) 721 [hep-ph/0501115].

[15] E. R. Nocera et al. [NNPDF Collaboration], A first unbiased global determination of polarized PDFs and their uncertainties, Nucl. Phys. B 887 (2014) 276 [arXiv:1406.5539].

[16] R. D. Ball et al. [The NNPDF Collaboration], Unbiased determination of polarized parton distributions and their uncertainties, Nucl. Phys. B 874 (2013) 36 [arXiv:1303.7236].

[17] J. Kodaira, S. Matsuda, T. Muta, K. Sasaki and T. Uematsu, QCD Effects in Polarized Electroproduction, Phys. Rev. D 20 (1979) 627.

[18] J. Kodaira, S. Matsuda, K. Sasaki and T. Uematsu, QCD Higher Order Effects in Spin Dependent Deep Inelastic Electroproduction, Nucl. Phys. B 159 (1979) 99.

[19] J. Kodaira, QCD Higher Order Effects in Polarized Electroproduction: Flavor Singlet Coefficient Functions, Nucl. Phys. B 165 (1980) 129.

[20] R. L. Jaffe, The Axial Anomaly and the Sum Rules for Spin Dependent Electroproduction, Phys. Lett. B 193 (1987) 101.

[21] A. V. Efremov and O. V. Teryaev, Spin Structure of the Nucleons and Triangle Anomaly, JINR Report E2-88-287, In *Prague 1988, Proceedings, Hadron Interactions Theory and Phenomenology* pp.302-312.

[22] G. Altarelli and G. G. Ross, The Anomalous Gluon Contribution to Polarized Leptoproduction, Phys. Lett. B 212 (1988) 391.

[23] R. D. Carlitz, J. C. Collins and A. H. Mueller, The Role of the Axial Anomaly in Measuring Spin Dependent Parton Distributions, Phys. Lett. B 214 (1988) 229.

[24] G. Altarelli and G. Parisi, Asymptotic Freedom in Parton Language, Nucl. Phys. B 126 (1977) 298.

[25] G. Altarelli and B. Lampe, The Light Cone Expansion and the Parton Model in Polarized Muon Production, Z. Phys. C 47 (1990) 315.

[26] Y. L. Dokshitzer, V. A. Khoze, A. H. Mueller and S. I. Troian, Basics of perturbative QCD, Gif-sur-Yvette, France: Ed. Frontieres (1991) pp 65-88.

[27] S. L. Adler, Axial vector vertex in spinor electrodynamics, Phys. Rev. 177 (1969) 2426.

[28] J. S. Bell and R. Jackiw, A PCAC puzzle: $\pi_{0}$ to $\gamma \gamma$ in the sigma model, Nuovo Cim. A 60 (1969) 47.

[29] A. V. Efremov, J. Soffer and O. V. Teryaev, Spin Structure of Nucleon and the Axial Anomaly, Nucl. Phys. B 346 (1990) 97.

[30] F. E. Close and R. G. Roberts, Consistent analysis of the spin content of the nucleon, Phys. Lett. B 316 (1993) 165 [hep-ph/9306289].

[31] F. E. Close, On the Transformation Between Current and Constituent Quarks and Consequences for Polarized Electroproduction Structure Functions, Nucl. Phys. B 80 (1974) 269.

[32] S. A. Larin, T. van Ritbergen and J. A. M. Vermaseren, The Alpha-s**3 approximation of quantum chromodynamics to the Ellis-Jaffe sum rule, Phys. Lett. B 404 (1997) 153.

[33] S. D. Bass and A. W. Thomas, The Nucleon's octet axial-charge $g(A) * *(8)$ with chiral corrections, Phys. Lett. B 684 (2010) 216 [arXiv:0912.1765 [hep-ph]].

[34] V. Y. Alexakhin et al. [COMPASS Collaboration], The Deuteron Spin-dependent Structure Function $g_{1}^{d}$ and its First Moment, Phys. Lett. B 647 (2007) 8 [hep-ex/0609038]. 
[35] B. Lampe, A Short review and some new results on polarization, Nucl. Phys. Proc. Suppl. 54A (1997) 134 [hep-ph/9608400].

[36] D. J. Gross and F. Wilczek, Asymptotically Free Gauge Theories. 2., Phys. Rev. D 9 (1974) 980. 